\title{
ORBITAL NOISE IN THE EARTH SYSTEM AND CLIMATE FLUCTUATIONS
}

\author{
Han-Shou Liu \\ Geodynamics Branch \\ NASA Goddard Space Flight Center \\ Greenbelt, Maryland 20771 USA
}

\begin{abstract}
Frequency noise in the variations of the Earth's obliquity (tilt) can modulate the insolation signal for climate change. Including this frequency noise effect on the incoming solar radiation, we have applied an energy balance climate model to calculate the climate fluctuations for the past one million years. Model simulation results are in good agreement with the geologically observed paleoclimate data. We conclude that orbital noise in the Earth system may be the major cause of the climate fluctuation cycles.

\section{INTRODUCTION}

The cause of the 100-Kyr ice age cycles during the past one million years has been a scientific mystery despite decades of studies. Although the Earth's orbital eccentricity signal varies with a $100-\mathrm{Kyr}$ cycle, the resulting changes in solar heating are too small to be climatically significant. How can such a strong climate response arise from such a seemingly weak forcing? Numerous hypotheses and models in terms of internal feedbacks such as ocean circulations and atmospheric $\mathrm{CO}_{2}$ concentrations have been proposed to understand this mystery. However, the origin of the climate fluctuations remains a perplexing and enduring problem. The purpose of this paper is to demonstrate that the cause of the major climate fluctuation cycles is mainly due to the frequency noise in the Earth's obliquity variations, which is distinct from the commonly considered signals in the orbital eccentricity and internal feedbacks.
\end{abstract}

\section{CLIMATE RESPONSE TO ORBITAL NOISE}

Climate response to insolation signal is intrinsically nonlinear in a noise environment of the Earth's orbital motion, including variations in eccentricity, obliquity and precession. The insolation amplitude signal alone, which is due to orbital variations, is unable to reproduce major spectral peaks as seen in the observed climate records. However, noise from the frequency variations in the obliquity may amplify the small signal of insolation forcing and cause the climate to fluctuate from glacial to interglacial conditions. The frequency noise from the obliquity is generated by the solar-luni and planetary gravitational torques exerted on the equatorial bulge of the Earth [1-6].

\section{NOISE-SIGNAL COOPERATIVE PHENOMENON}

Based on the convolution theory of the non-stationary time series of orbital motion, the frequency noise in the obliquity can be expressed by $[4,5]$. 


$$
N(t)=\frac{1}{\omega_{c}} \frac{\mathrm{d}}{\mathrm{dt}}\left\{\tan ^{-1}\left[\frac{\sum_{i=1} \mathrm{~A}_{\mathrm{i}} \sin \left(\gamma_{\mathrm{i}} \mathrm{t}+\zeta_{\mathrm{i}}\right)}{\sum_{\mathrm{i}=1} \mathrm{~A}_{\mathrm{i}} \cos \left(\gamma_{\mathrm{i}} \mathrm{t}+\zeta_{\mathrm{i}}\right)}\right]\right\}
$$

where the amplitude $A_{t}$, frequency $\gamma_{1}$ and phase $\xi_{\mathrm{r}}$ for $i=1,2,3 \ldots .80$ are astronomical constants [7]. Liu [2-5] and Liu and Chao [6] have shown that the signal of the incoming solar radiation has been embedded in the frequency noise $N(t)$ of the obliquity. This insolation signal $S(t)$ is governed by [8].

$$
S(t)=\Delta R_{s} \Delta \varepsilon(t)+m \Delta(e \sin \omega)
$$

where $\Delta R_{s}$ is the difference of the insolation received at the top of the atmosphere for $1^{\circ}$ change in obliquity $\in(t), e \sin \varpi$ is the precession variation and $m$ is a function of the duration of the tropical year, the orbital eccentricity and the latitude. The cooperative phenomenon between the signal $S(t)$ and noise $N(t)$ is shown in Fig $1 a$ for the past one million years in which the two

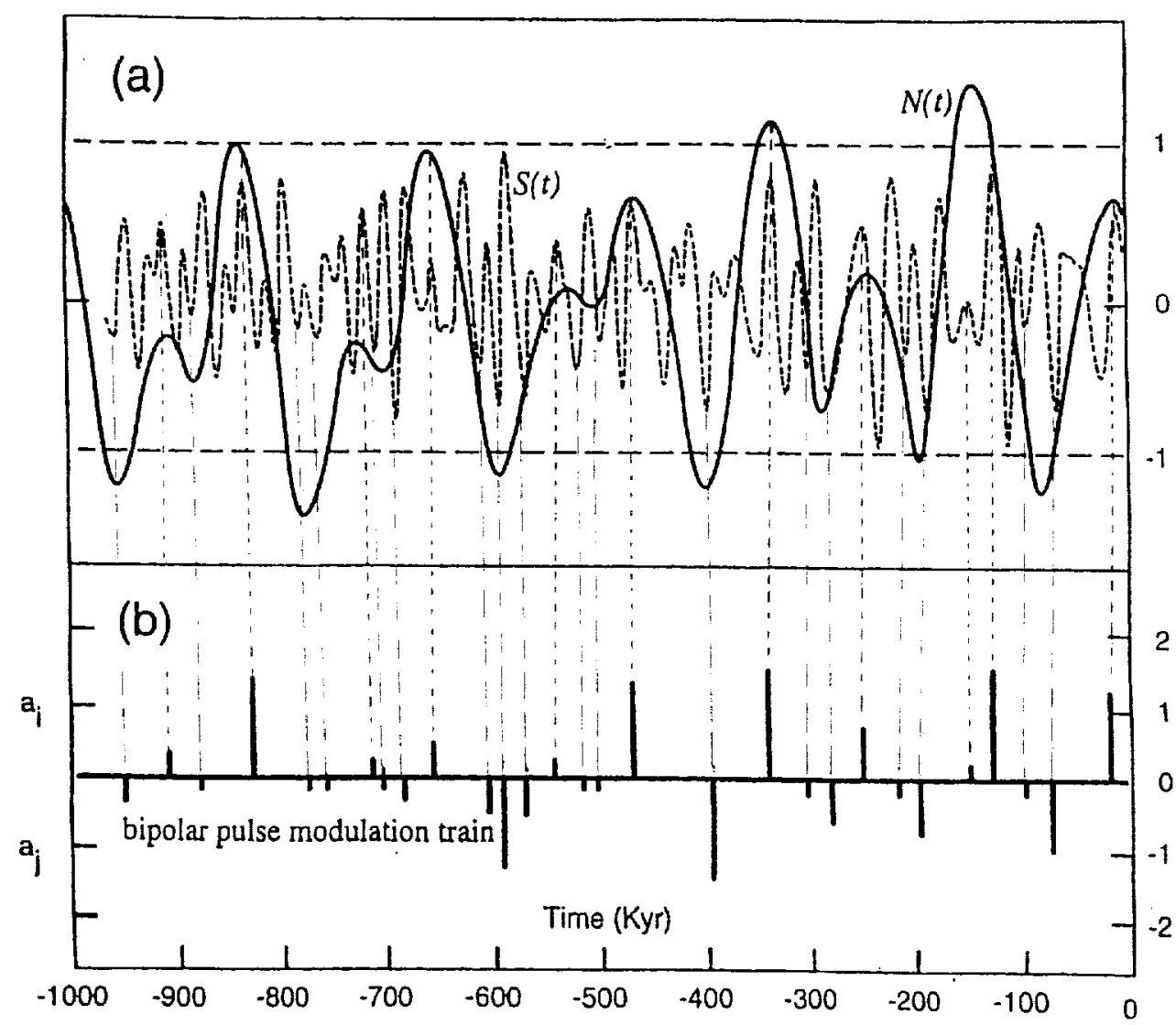

Figure 1. Cooperative phenomenon between insolation signal $S(t)$ and frequency noise $N(t)$ of the Earth's obliquity variation. 
dashed threshold lines for $S(t)$ represent maximum and minimum values of insolation deviation at latitude $65^{\circ} \mathrm{N}$. Hence, amplitudes of $S(t)$ are subthreshold [9]. To scale $N(t)$ as a pulse function, the pulse magnitudes (length of the bars in Fig $1 b$ ) are chosen such that they can be normalized between \pm 2 with zero mean. Fig $1 b$ shows a bipolar pulse modulation train of the insolation. Each time the magnitude of $S(t)$ plus its corresponding $N(t)$ crosses the threshold a pulse can be written to the time series of the insolation. It has been found that the timing of the insolation pulses coincides with the occurrence of major paleoclimate changes [4,5]. The pulse modulation train for the insolation signal $S(t)$ as shown in Fig $1 b$ can be expressed by [10]

$$
c(t)=\sum_{i} a_{i} P\left(t-t_{i}\right)-\sum_{j} a_{j} P\left(t-t_{j}\right)
$$

where $a_{l}$ and $a_{j}$ are the magnitude of the insolation pulses and $t_{l}$ and $t_{j}$ are time indices of rapid melting and prolonged glaciation.

\section{CLIMATE FLUCTUATION}

The noise-signal cooperative phenomenon may cause pulses in the insolation signal $S(t)$. Including this pulse modulation effect, equation of the O-D energy balance climate model becomes [3-5]

$$
\begin{gathered}
c \frac{d T}{d t}=S(t)\left[1+\sum_{i} a_{i} P\left(t-t_{i}\right)-\sum_{j} a_{j} P\left(t-t_{j}\right)\right] \\
\cdot\left\{1-d_{1}+d_{2} \tanh \left[d_{3}\left(T-T_{0}\right)\right]\right\}-A-B T
\end{gathered}
$$

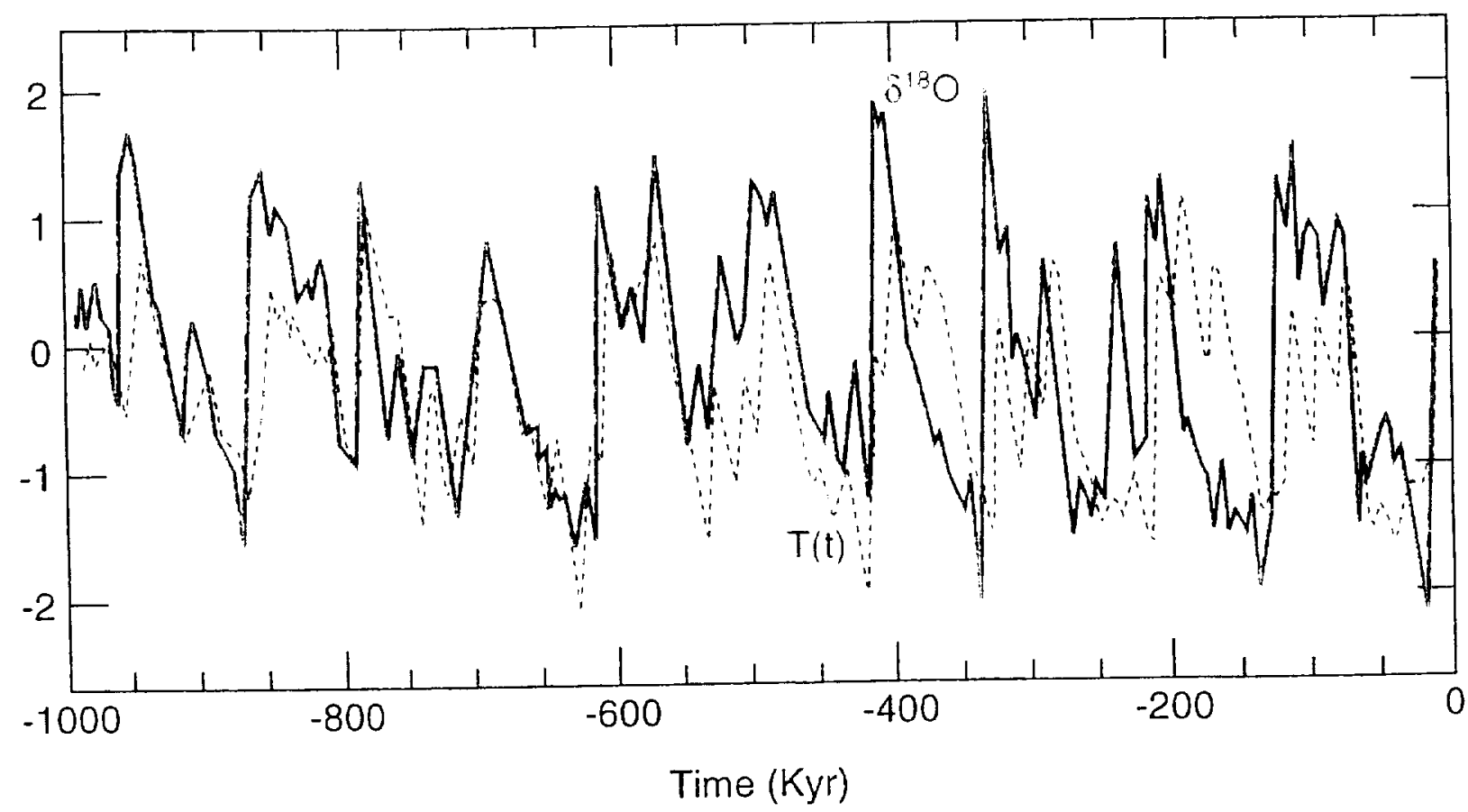

Figure 2. Temperature fluctuation simulation $T(t)$ from model equation (4) and the oxygen isotop $\delta^{18} 0$ concentration variations from [12]. 
where $d_{l,} d_{2,} d_{3} T_{0}, \mathrm{~A}$ and $\mathrm{B}$ are geophysical constants [11]. We have performed a series of computations to test model response to the pulse-modulated insolation. The fluctuation of the Earth's surface temperature $T(t)$ in equation (4) for latitude $65^{\circ} \mathrm{N}$ 'is shown in Fig. 2, in good agreement with variations in the oxygen isotop content $\delta^{l 8} 0$ of deep-sea sedimentary cores as obtained by [12]. Extension of model simulation reveals that the current warming trend of the climate is almost over and will give way to forecast for a small ice age.

\section{DISCUSSSION AND CONCLUSION}

A faithful representation of climate fluctuations in computer form is needed to clarify events of the past, better to predict the future. But the route to that end still lies ahead of us because the cause of the major climate fluctuation cycles remains a scientific mystery today despite decades of investigation. This paper has revolutionized the knowledge of orbital dynamics of the Earth system and climatology. It has been found that climate response to insolation signal is intrinsically nonlinear in a noisy environment of orbital motion. The insolation amplitude signal alone, which is due to orbital variations, is unable to reproduce major spectral peaks as seen in the proxy climate data. The frequency noise in the Earth's obliquity, however, may produce a noise-signal cooperative phenomenon that amplifies the small insolation forcing. This new forcing mechanism could enhance the probability of jumping between the glacial and interglacial climate states.

\section{ACKNOWLEDGMENTS}

The author thanks Clarence Wade Jr. at the Laboratory for Astronomy and Solar Physics and Dereck L. Ho at the National Standard and Technology for computation assistance.

\section{REFERENCES}

[1] H.S. Liu, Nature, 358, pp.397-399 (1992).

[2] H.S. Liu, Earth and Planetary Sci. Lett., 131, pp. 17-26 (1995).

[3] H.S. Liu, Theor. Appl. Climatol., 61, pp. 217-229 (1998).

[4] H.S. Liu, J. Geophy. Res. - Atmosphere, 103, D20, pp. 26,147-164 (1998).

[5] H.S. Liu, J. Geophy. Res. - Solid Earth, 104, B11, pp. 25,197-206 (1999).

[6] H.S. Liu and B.F. Chao, J. Atmosphere Sci., 55, 2, pp. 227-236 (1998).

[7] J. Laskar, Astro, Astophys., 198, pp. 341-361 (1998).

[8] A.D. Vernekar, Meteor. Monogr. 34, Amer. Meteor. Soc., pp. 1-21 (1972).

[9] L. Gammaitoni, Phys. Rev. E., 52, pp. $4691-4698$ (1995).

[10] H.E. Rowe, Signal and Noise in Communication System, Van Nostand Reinhold, New York, 341 (1965).

[11] G. Matteucci, Climate Dynamics, 3, pp. 179-190 (1989).

[12] N.J. Shackleton, et Al., Trans. R. Soc., Edinburgh Earth Sci. Rev., pp. 251-261 (1990). 